\title{
Mesh Refinements for Parabolic Equations of Second Order*
}

\author{
By Stewart Venit
}

\begin{abstract}
Given certain implicit difference approximations to $u_{t}=a(x) u_{x x}+b(x) u_{x}+$ $c(x) u$ in the region $-\infty<x<\infty, t \geqq 0$, with a finer $x$-mesh width in the left half-plane than in the right, we consider the stability in the maximum norm of these schemes using several different interface conditions (at $x=0$ ). In order to obtain our results, we first prove a stability theorem for certain simple second-order parabolic initial boundary systems on an evenly spaced mesh in the right half-plane alone. By a standard procedure, the first problem is converted into the second one, and solved in this manner.
\end{abstract}

0. Introduction. In [2], Osher shows the stability in the maximum norm of certain finite-difference approximations to $u_{t}=u_{x x}$, where the mesh width is finer in the left half-plane than in the right. It is the object of this paper to generalize the above result to approximations for parabolic second-order equations with variable coefficients, using various types of "interface conditions."

Following a standard procedure, this stability problem is converted into an equivalent one concerning a system of two difference equations on a uniform mesh in the right half-plane alone. The latter is solved by partially generalizing a theorem of Osher [3] dealing with the stability of parabolic difference equations of the initial boundary type.

The original stability problem is generally referred to as a "mesh refinement problem." These have been treated by Ciment [1] and Varah [7], as well as by Osher. The work presented here is mostly taken from the author's Ph.D. thesis, where it is given in much more detail, but in the case of the two mesh refinement theorems of Section 4, in somewhat less general form.

I would like to thank Professor Stanley Osher for offering many valuable suggestions, as well as guidance and encouragement, toward the completion of the thesis upon which this work is based.

1. The Mesh Refinement Problem. We will approximately solve the initialvalue problem

$$
\begin{gathered}
u_{t}=a(x) u_{x x}+b(x) u_{x}+c(x) u, \quad-\infty<x<\infty, \quad 0<t \leqq T<\infty . \\
u(x, 0)=f(x), \quad-\infty<x<\infty,
\end{gathered}
$$

Received June 30, 1972, revised December 18, 1972.

AMS (MOS) subject classifications (1970), Primary 65M10.

Key words and phrases. Difference scheme, stability, mesh refinement, parabolic partial differential equation, initial boundary-value problem.

* This research was supported in part by the Office of Naval Research under contract number N00014-69-A-0200-0017. 
on a mesh with nodes at

$$
\begin{aligned}
t_{n} & =n k, \quad n=0,1, \cdots, T / k ; \\
x_{i} & =j h, \quad j=0,1,2, \cdots ; \quad x=j h / p, \quad j=-1,-2, \cdots .
\end{aligned}
$$

The coefficients in (1.1) are to satisfy the following:

$$
\begin{aligned}
& a(x) \geqq \text { const }>0,\left|a(x)-a\left(x_{0}\right)\right| \leqq \text { const }\left|x-x_{0}\right|^{\gamma} \text { with } \\
& \gamma>0, \text { and } b(x), c(x), \text { and } f(x) \text { bounded and measurable. }
\end{aligned}
$$

The difference scheme is of the form:

$$
\sum_{i=-1}^{1} b_{i}(x, h) v(x+i h, t+k)=\sum_{i=-1}^{1} c_{i}(x, h) v(x+i h, t)
$$$$
\text { for } x=h, 2 h, 3 h, \cdots \text {, }
$$

$$
\begin{aligned}
& \sum_{i=-1}^{1} b_{i}^{\prime}(x, h) v(x+i h / p, t+k)=\sum_{i=-1}^{1} c_{i}^{\prime}(x, h) v(x+i h / p, t) \\
& \text { for } x=-h / p,-2 h / p,-3 h / p, \cdots \text {, } \\
& \sum_{i=1}^{s} p_{i}(h) v(i h, t+k)+\sum_{i=-r}^{0} p_{i}(h) v(i h / p, t+k)
\end{aligned}
$$$$
=\sum_{i=1}^{s} q_{i}(h) v(i h, t)+\sum_{i=-r}^{0} q_{i}(h) v(i h / p, t) \text { for } x=0,
$$

In (1.3)-(1.5), $t=0, k, 2 k, \cdots, T$, and $r, s$, and $p$ are positive integers. Restrictions on the coefficients will be given in Assumptions (i)-(iv).

Remark. Notice that we are only considering "three-point" schemes; that is, the values of $v$ are needed at only three points on each time level in Eqs. (1.3) and (1.4). (The "interface condition," Eq. (1.5), can be of a more general nature.) In his Ph.D. thesis [8], the author also considers $(2 r+1)$-point schemes, but these are limited to constant coefficients. Moreover, the applications given mainly concern the three-point variety, so for the sake of brevity, these are the only ones considered in this paper.

Assumption (i). Equations (1.3) and (1.4) are consistent with (1.1).

Assumption (ii). The coefficients $b_{i}(x, 0), c_{i}(x, 0), b_{i}^{\prime}(x, 0)$, and $c_{i}^{\prime}(x, 0)$ are bounded and uniformly Hölder continuous with $b_{-1}(x, 0) \neq c_{-1}(x, 0)$ and $b_{1}(x, 0) \neq c_{1}(x, 0)$. Also, the coefficients $p_{i}(h)$ and $q_{i}(h)$ are $C^{2}(h)$ for $h$ small.

Assumption (iii). Equations (1.3) and (1.4) are parabolic; that is, there exists $\delta>0$ such that if $z$ is a root of

$$
\sum_{i=-1}^{1}\left(c_{i}(x, 0)-z b_{i}(x, 0)\right) e^{i j \theta}=0,
$$

then $z$ satisfies $|z| \leqq 1-\delta \theta^{2}$, for all $|\theta| \leqq \pi$ (and a similar statement holds for (1.4)).

Assumption (iv). Equations (1.3)-(1.5) are invertible; that is, for arbitrary righthand sides in these expressions, there exists a unique $v(x, t+k)$ satisfying the equations with $\|v(\cdot, t+k)\|$ bounded by a constant times the norm of the right-hand side. (I| || will always denote the maximum norm.) 
Necessary and sufficient conditions for the invertibility of different schemes are given by Osher in [3] and [4].

We also assume, merely for the sake of convenience, that (1.3) and (1.4) are symmetric; that is, $b_{-1}(x, 0)=b_{1}(x, 0), c_{-1}(x, 0)=c_{1}(x, 0)$, and the same holds for the "primed" coefficients.

2. A Stability Theorem for the Equivalent Initial Boundary System. If we introduce the two-component vector

$$
w(x, t)=(v(x, t), v(-x / p, t))^{\prime},
$$

Eqs. (1.3)-(1.6) are converted into an equivalent initial boundary system in the right half-plane.

$$
\begin{aligned}
& \sum_{i=-1}^{1} B_{i}(x, h) T^{i} w(x, t+k)=\sum_{i=-1}^{1} C_{i}(x, h) T^{i} w(x, t) \\
& \quad \text { for } x=h, 2 h, 3 h, \cdots ; t=0, k, 2 k, \cdots, T,
\end{aligned}
$$

where

$$
\begin{aligned}
& T w(x, t)=w(x+h, t), F(x)=(f(x), f(-x / p))^{\prime}, \\
& B_{i}(x, h)=\left|\begin{array}{cc}
b_{i}(x, h) & 0 \\
0 & b_{i}^{\prime}(-x / p, h)
\end{array}\right|, \quad C_{i}(x, h)=\left|\begin{array}{cc}
c_{i}(x, h) & 0 \\
0 & c_{i}^{\prime}(-x / p, h)
\end{array}\right|
\end{aligned}
$$

and the $P_{i}$ and $Q_{i}$ are square matrices of order two. The first row of all $P_{i}$ and $Q_{i}$ except $P_{0}$ have zero entries, while the first row of $P_{0}$ has entries 1 and -1 .

We now proceed to state a stability theorem for $(2.1)-(2.3)$. The hypotheses of this theorem will include conditions to insure the stability of the related pure initialvalue problem, as well as restrictions on the boundary conditions (2.3). The latter are given in terms of a "boundary matrix" similar to the one employed by Varah [6], who only considered boundary conditions on one time level.

Consider the pair of equations in the vector unknown, $g(j h, n k)$ :

$$
\begin{gathered}
\sum_{i=-1}^{1}\left(C_{i}(0,0)-z B_{i}(0,0)\right) g((i+j) h, n k)=0, \quad j=1,2,3, \cdots, \\
\sum_{i=0}^{l}\left(Q_{i}(h)-z P_{i}(h)\right) g(j h, n k)=0 .
\end{gathered}
$$

For $|z| \geqq 1, z \neq 1$, it can be shown (using the parabolicity of the scheme) that the following quadratic equations have no root $x$ with $|x|=1$ :

$$
\begin{aligned}
& \sum_{i=-1}^{1}\left(c_{i}(0,0)-z b_{i}(0,0)\right) x^{i+1}=0, \\
& \sum_{i=-1}^{1}\left(c_{i}(0,0)-z b_{i}(0,0)\right) x^{i+1}=0 .
\end{aligned}
$$


Consequently, Eq. (2.4) has the solution

$$
g(j h, n k)=\left(d_{1} t_{1}^{i}, d_{2} t_{2}^{i}\right)^{\prime},
$$

where $d_{1}$ and $d_{2}$ are arbitrary constants, and $t_{1}$ and $t_{2}$ are functions of $z$ satisfying (2.6) and (2.7), respectively, and having modulus less than one for $|z| \geqq 1, z \neq 1$. Substituting this expression for $g$ into (2.5) yields two linear homogeneous equations in the parameters $d_{1}$ and $d_{2}$. Letting $\mathrm{d}=\left(d_{1}, d_{2}\right)^{\prime}$, we can write these last equations as

$$
E(z, h) \mathrm{d}=0
$$

where $E(z, h)$ is called the boundary matrix for the scheme. Explicitly, $E(z, h)$ is given by

$$
E(z, h)_{j k}=\sum_{i=0}^{l}\left(Q_{i}(h)-{ }_{z} P_{i}(h)\right)_{j k}\left(t_{k}\right)^{i} \quad \text { with } j, k=1,2 .
$$

THEOREM (2.1). Given the difference scheme (2.1)-(2.3), suppose that

(a) Assumptions (i)-(iv) of Section 1 are satisfied,

(b) $\operatorname{det} E(z, 0) \neq 0$ for $|z| \geqq 1, z \neq 1$,

(c) $\operatorname{det} E(z, 0)=O\left((z-1)^{1 / 2}\right)$ as $z \rightarrow 1,|z| \geqq 1$.

Then, the scheme is stable in the maximum norm; that is, there exists $K=K(T)$, independent of $n, k$, and $h$, such that

$$
\|w(\cdot, n k)\| \equiv \max _{i=1,2, \cdots}|w(j h, n k)| \leqq K\|F\| .
$$

3. Proof of Theorem (2.1). The hypotheses in Theorem (2.1) are essentially those of Varah's stability theorem in [6]. The latter, however, requires consistency of the boundary conditions to insure stability, while this is shown to be unnecessary for three-point variable coefficient schemes in [3], as well as for $(2 r+1)$-point constant coefficient schemes in [8]. Of course, to obtain convergence by the Lax-Richtmyer theorem, we do need consistency, but not requiring it at this point is convenient in proving mesh refinement theorems.

The proof closely follows that of Osher [3], who in turn made use of techniques of Varah [6] and Widlund [9]. Ours differs in some respects due to the aforementioned lack of consistency at the boundary, the "linking" of the two equations in (2.1)-(2.3) at the boundary, and the use of two level boundary conditions. For this reason, we outline most of the proof, referring the reader to Osher [3] or Venit [8] for more detail, and fill in the detail ourselves concerning the above points.

Our goal is to obtain estimates on the zeroth, first, and second divided differences of

$$
G\left(j h, n k, j_{0} h\right)=-\frac{1}{2 \pi i} \int_{S} z^{n}\left(V\left(j, z, j_{0}\right)+W\left(j, z, j_{0}\right)\right) d z
$$

where $V_{i}$ and $W_{i}$ are solutions of Eqs. (3.1)-(3.3) below. Once these estimates are obtained, the parametrix technique of Widlund [9] may be employed. The details of this procedure are as given by Osher in [3].

$$
\begin{aligned}
& \sum_{i=-1}^{1}\left(C_{i}\left(x_{0}, 0\right)-z B_{i}\left(x_{0}, 0\right)\right) V_{j+i}=\delta(i, j) I, \\
& x_{0}=j h, j-j_{0}=0, \pm 1, \pm 2, \cdots ; \delta \text { is the Kronecker } \\
& \delta \text {, and } I \text { is the second-order identity matrix, }
\end{aligned}
$$




$$
\begin{gathered}
\sum_{i=-1}^{1}\left(C_{i}(0,0)-z B_{i}(0,0)\right) W_{i+i}=0, \quad j=1,2,3, \cdots, \\
\sum_{i=0}^{l}\left(Q_{i}(h)-z P_{i}(h)\right)\left(V_{i}+W_{i}\right)=0 .
\end{gathered}
$$

$S=S_{1}+S_{2}+S_{3}$ is the contour defined in Osher [3], and is given below:

$$
\begin{array}{ll}
S_{1}:|z|=e^{-\delta}, \quad|\arg z| \geqq \theta_{1}^{2}, & \\
S_{2}: z=\exp \left(K i \theta^{2}-\theta^{2}+\beta^{2} h^{2}\right), & 0 \leqq \arg z \leqq \theta_{1}^{2}, \\
S_{3}: z=\exp \left(K i \theta^{2}-\theta^{2}+\beta^{2} h^{2}\right), & -\theta_{1}^{2} \leqq \arg z \leqq 0 .
\end{array}
$$

Here, $\theta_{1}^{2}=\arg z_{0}$, where $z_{0}$ is the point of intersection of $S_{1}$ and $S_{2}$, and $K, \delta$, and $\beta$ are defined in the course of the proof.

Estimates are obtained on $V$ and $W$ in two regions. One includes $S_{1}$, and the other includes $S_{2}$ and $S_{3}$. We will simply say that our estimates hold "on $S_{1}$ " or "on $S_{2}$ and $S_{3}$," or, equivalently, "away from $z=1$ " or "near $z=1$."

Near $z=1$, Eqs. (2.6) and (2.7) have the solutions (respectively)

$$
t_{1}(z)=1-\left(\frac{z-1}{\lambda a(0)}\right)^{1 / 2}+O(z-1), \quad t_{2}(z)=1-\left(\frac{z-1}{\lambda p^{2} a(0)}\right)^{1 / 2}+O(z-1),
$$

where the branch cut for the square root is taken from $-\infty$ to 1 along the $x$ axis, and $\left|t_{1}(z)\right|<1,\left|t_{2}(z)\right|<1$.

Away from $z=1$, these roots are bounded away from $\left|t_{i}\right|=1$.

The above, together with (3.1), gives us the following expressions for $V\left(j, z, j_{0}\right)$. On $S_{2}$ or $S_{3}$, for $\left|j-j_{0}\right|=0,1,2, \cdots$,

$$
V_{i k}=\left(\delta(i, k) g_{i}(z)\left(t_{i}\left(x_{0}, z\right)\right)^{\left|i-i_{0}\right|}\right) /(z-1)^{1 / 2} .
$$

Here, the $g_{i}(z)$ are analytic functions of $(z-1)^{1 / 2}$, and the $t_{i}\left(x_{0}, z\right)$ satisfy (2.6) or (2.7) with coefficients evaluated at $\left(x_{0}, 0\right)$ instead of $(0,0)$.

On $S_{1}$, for $\left|j-j_{0}\right|=0,1,2, \cdots$,

$$
\left|V_{i k}\right| \leqq \delta(i, k) K_{1} \rho^{\left|i-i_{0}\right|}, \quad \rho<1, K_{1}=\text { constant }
$$

(In (3.4), (3.5) the indices $i, k=1,2$.)

Since (3.2) is a homogeneous linear difference equation, for $j=1,2,3, \cdots$,

$$
W_{i k}=d_{i k}\left(t_{i}(z)\right)^{i} \quad(i, k=1,2) .
$$

Substituting this into (3.3), and defining the matrix $D$ by $D_{i k}=d_{i k}(i, k=1,2)$ yields

$$
-E(z, h) D=\sum_{i=0}^{l}\left(Q_{i}(h)-P_{i}(h)\right) V\left(j, z, j_{0}\right) .
$$

Since det $E(z, 0) \neq 0$ for $|z| \geqq 1, z \neq 1$, it can be shown that det $E(z, h) \neq 0$ for $z$ on $S_{1}$, and thus, by using (3.5), (3.6), and (3.7), we obtain the following estimate for $z$ on $S_{1}, i, j=1,2$ :

$$
\left|W_{i k}\right| \leqq K_{1} \rho^{\left|i-i_{0}\right|}, \quad \rho<1, K_{1}=\text { constant } .
$$

To estimate $W$ on $S_{2}$ and $S_{3}$, we define the following second-order matrices 
$(m, n=1,2)$ :

$$
\begin{aligned}
{\left[A_{i}\right]_{m, n} } & =\delta(m, n)\left(g_{m}(z)\left(t_{m}\left(x_{0}, z\right)\right)^{i_{0}-i}\right) /(z-1)^{1 / 2}, \\
{\left[A_{i}^{\prime}\right]_{m, n} } & =\delta(m, n)\left(g_{m}(z)\left(t_{m}\left(x_{0}, z\right)\right)^{i-i_{0}}\right) /(z-1)^{1 / 2}, \\
T_{m, n} & =\delta(m, n) t_{m}\left(x_{0}, z\right) .
\end{aligned}
$$

Then, we can write (3.7), with the aid of (3.4), as

$$
\begin{aligned}
& -E(z, h) D=\sum_{i=0}^{l}\left(Q_{i}(h)-z P_{i}(h)\right) A_{i}+\sum_{i=i_{0}}^{l}\left(Q_{i}(h)-z P_{i}(h)\right)\left(A_{i}^{\prime}-A_{i}\right) \\
& =\sum_{i=0}^{l}\left(Q_{i}(h)-z P_{i}(h)\right)\left(T^{i}+\left(T^{-i}-T^{i}\right)\right) A_{0}+\sum_{i=i_{0}}^{l}\left(Q_{i}(h)-z P_{i}(h)\right)\left(A_{i}^{\prime}-A_{i}\right) .
\end{aligned}
$$

Now, $\sum_{i=0}^{l}\left(Q_{i}(h)-z P_{i}(h)\right) T^{i}-E(z, h)=O\left((z-1)^{1 / 2}\right)$, and $T^{-i}-T^{i}=$ $O\left((z-1)^{1 / 2}\right)$. Hence, there exist $F_{1}(z, h)$ and $F_{2}\left(j_{0}, z, h\right)$, both analytic in $(z-1)^{1 / 2}$ with $F_{2}\left(j_{0}, z, h\right)=0$ if $j_{0}>l$, such that

$$
-E(z, h) D=\left(E(z, h)+(z-1)^{1 / 2} F_{1}\right) A_{0}+F_{2} .
$$

Now, $E(z, h)^{-1}$ exists on $S_{2}$ and $S_{3}$ (see Osher [3]). Therefore,

$$
D=-\left(I+\frac{(z-1)^{1 / 2} F_{3}(z, h)}{\operatorname{det} E(z, h)}\right) A_{0}+\frac{F_{4}\left(j_{0}, z, h\right)}{\operatorname{det} E(z, h)},
$$

where $F_{3}$ and $F_{4}$ have the same properties as $F_{1}$ and $F_{2}$, respectively. Combining this last equation with (3.6) gives the following estimate for $z$ on $S_{2}$ and $S_{3}, j=0,1,2, \cdots$,

$$
W_{i k}=\left[-\frac{g_{i}(z)\left(t_{k}\left(x_{0}, z\right)\right)^{i}}{(z-1)^{1 / 2}}\left(\delta(i, k)+\frac{F_{1}(z, h)_{i k}(z-1)^{1 / 2}}{\operatorname{det} E(z, h)}\right)+\frac{F_{2}\left(j_{0}, z, h\right)_{i k}}{\operatorname{det} E(z, h)}\right]\left(t_{i}(z)\right)^{i} .
$$

It can be shown that det $E(z, h)=\left((z-1)^{1 / 2}+c h\right) f(z, h)$, where $f$ and $f^{-1}$ are analytic functions of $(z-1)^{1 / 2}$.

The proof is then completed by noticing that (3.4), (3.5), (3.8), and (3.9) give us estimates for each entry of the divided differences of $G\left(j h, n k, j_{0} h\right)$ which are similar to those of Osher in [3]. We can therefore proceed in the same manner to obtain the desired stability inequality. (For more detail here, also see Venit [8].)

4. Mesh Refinement Theorems. First, we will extend a theorem due to Osher given in [2].

THEOREM (4.1). Let (1.3), (1.4), (1.6) approximate (1.1) on the mesh (1.2) with the interface condition

$$
\sum_{i=-1}^{1} b_{i}(0, h) v(i h, t+k)=\sum_{i=-1}^{1} c_{i}(0, h) v(i h, t) .
$$

Assume that the scheme is smooth, consistent, parabolic, symmetric, and invertible. Then, it is stable in the maximum norm.

Remark. An interface condition of this type is known as "using the difference equation itself at the interface."

Proof. Introduction of the vector $w(x, t)=(v(x, t), v(-x / p, t))^{\prime}$ converts this 
scheme into an initial boundary one of the type (2.1)-(2.3) with

$$
\begin{aligned}
& P_{0}=\left|\begin{array}{cc}
1 & -1 \\
b_{0}(0, h) & 0
\end{array}\right|, \quad P_{1}=\left|\begin{array}{cc}
0 & 0 \\
b_{1}(0, h) & 0
\end{array}\right|, \quad P_{p}=\left|\begin{array}{cc}
0 & 0 \\
0 & b_{1}(0, h)
\end{array}\right|, \\
& Q_{0}=\left|\begin{array}{cc}
0 & 0 \\
c_{0}(0, h) & 0
\end{array}\right|, \quad Q_{1}=\left|\begin{array}{cc}
0 & 0 \\
c_{1}(0, h) & 0
\end{array}\right|, \quad Q_{p}=\left|\begin{array}{cc}
0 & 0 \\
0 & c_{1}(0, h)
\end{array}\right| \text {. }
\end{aligned}
$$

All other $Q_{i}$ and $P_{i}$ are zero. We now verify conditions (b) and (c) of Theorem (2.1). Letting $b_{i}=b_{i}(0,0)$ and $c_{i}=c_{i}(0,0)$, we have

$$
E(z, 0)=\left|\begin{array}{cc}
z & -z \\
c_{0}-z b_{0}+\left(c_{1}-z b_{1}\right) t_{1} & \left(c_{1}-z b_{1}\right) t_{1}^{p}
\end{array}\right|,
$$

where $t_{1}$ satisfies (2.6), and consequently,

$$
c_{0}-z b_{0}+\left(c_{1}-z b_{1}\right) t_{1}=-\left(c_{1}-z b_{1}\right)\left(t_{1}^{p}-1 / t_{1}\right) .
$$

Hence, det $E(z, 0)=z\left(c_{1}-z b_{1}\right)\left(t_{1}^{p}-1 / t_{1}\right)$. If we assume that $z$ is such that $c_{1}-$ $z b_{1} \neq 0$, then since $\left|t_{1}\right|<1$ for $|z| \geqq 1, z \neq 1$, det $E(z, 0) \neq 0$ there. On the other hand, if $c_{1}-z b_{1}=0$, then $\operatorname{det} E(z, 0)=c_{0}-z b_{0}$. Suppose that $\operatorname{det} E(z, 0)=0$. Then,

$$
c_{1} b_{0}=c_{0} b_{1} \text {. }
$$

Without loss of generality, we may assume that $a(0)=1$. Then, consistency yields the equations

$$
2 c_{1}+c_{0}=2 b_{1}+b_{0}, \quad 2 c_{1}-2 b_{1}=2 \lambda .
$$

These two equations, together with (4.2) imply that $b_{0}+2 b_{1}=0$, which contradicts the invertibility of the scheme (see Osher [3], [4]). Hence, det $E(z, 0) \neq 0$ for any $z$ with $|z| \geqq 1, z \neq 1$.

Moreover, since $t_{1}=1-(z-1)^{1 / 2} /(\lambda a(0))^{1 / 2}+O(z-1)$ near $z=1$, det $E(z, 0)$ $=O\left((z-1)^{1 / 2}\right)$. Thus, we have verified the conditions of Theorem (2.1), as desired.

In the next theorem, for simplicity of notation, we assume that we are approximating $u_{t}=u_{x x}$ on the mesh (1.2). A similar theorem for approximations to (1.1) is easily proved in the same manner.

THEOREM (4.2). Given any consistent, parabolic, and invertible approximation to $u_{t}=u_{x x}$ on the mesh (1.2). Let the interface condition be of the form

$$
\begin{aligned}
b_{-1} v(-h / p, t+k) & +b_{0} v(0, t+k)+b_{1} v(h, t+k) \\
& =c_{-1} v(-h / p, t)+c_{0} v(0, t)+c_{1} v(h, t)
\end{aligned}
$$

where the coefficients are given by

$$
\begin{array}{ccc}
b_{-1}=-2 \lambda p^{2} \theta /(p+1), & b_{0}=1+2 \lambda p \theta, & b_{1}=-2 \lambda p \theta /(p+1), \\
c_{-1}=2 \lambda p^{2}(1-\theta) /(p+1), & c_{0}=1-2 \lambda p(1-\theta), & c_{1}=2 \lambda p(1-\theta) /(p+1) \\
& \left(0 \leqq \theta \leqq 1, \lambda=k / h^{2}\right) . &
\end{array}
$$

Then, the resulting scheme is stable in the maximum norm if $1-2 \lambda p(1-\theta) \geqq 0$ when $\theta<\frac{1}{2}$, and $\lambda>0$ otherwise. 
Remark. Notice that here the interface condition makes use of three successive points (on each time level), unlike (4.1), which skipped $p-1$ points in the fine mesh. The coefficients given above, as well as the restrictions placed upon them, are analogous to those for general two level three-point schemes with even mesh spacing (see Richtmyer and Morton [5, p. 189]).

Proof. Converting to an initial boundary system, we find that

$$
\text { det } \begin{aligned}
E(z) & =z\left(c_{0}-z b_{0}+\left(c_{1}-z b_{1}\right) t_{1}+\left(c_{-1}-z b_{-1}\right) t_{2}\right) \\
& =z\left[1-z+2 \lambda p(1-\theta+z \theta)\left(\frac{t_{1}+p t_{2}}{p+1}-1\right)\right] .
\end{aligned}
$$

Immediately, we see that det $E(z)=O\left((z-1)^{1 / 2}\right)$ near $z=1$. Now, suppose that $\operatorname{det} E(z)=0$ for some $z$ with $|z| \geqq 1, z \neq 1$. Then

$$
z=(1-2 \lambda p(1-\theta)(1-\tau)) /(1+2 \lambda p \theta(1-\tau)),
$$

where $\tau=\left(t_{1}+p t_{2}\right) /(p+1)$, and hence $|\tau|<1$.

If $1-2 \lambda p(1-\theta) \geqq 0$, then $|z| \leqq 1-2 \lambda p(1-\theta)+|2 \lambda p(1-\theta) \tau|<1$, a contradiction. If $1-2 \lambda p(1-\theta)<0$, we need only consider values of $\theta$ with $\frac{1}{2} \leqq \theta \leqq 1$. Here,

$$
|z|=\frac{|1-2 \lambda p(1-\theta)(1-\tau)|}{|1+2 \lambda p \theta(1-\tau)|} \leqq \frac{|1-\lambda p(1-\tau)|}{|1+\lambda p(1-\tau)|}<1 .
$$

The last inequality follows from a consideration of the real and imaginary parts of the quantities within the absolute value bars. Thus, once again we get a contradiction, and our theorem is proved.

An example of an interface condition which leads to an unstable scheme is

$$
v(0, t)=\sum_{i=1}^{m}(-1)^{i+1}\left(\begin{array}{c}
m \\
i
\end{array}\right) v(i h, t)
$$

where $\left(\begin{array}{c}m \\ i\end{array}\right)$ denote binomial coefficients. Notice that we are giving $v(0, t)$ in terms of values of $v$ taken at points lying only in the coarse mesh. If $m \geqq 2$, this equation is consistent with $u_{t}=u_{x x}$. Here, det $E(z)=\left(t_{1}-1\right)^{m}$, which is not equal to zero for $|z| \geqq 1, z \neq 1$. However, det $E(z)=O\left((z-1)^{m / 2}\right)$ near $z=1$, which violates condition (c) of Theorem (2.1) (if consistency holds). This itself does not prove instability, but lack of convergence for explicit three-point schemes employing this interface condition can easily be shown for the initial values: $f(x)=1$ if $x<0, f(x)=0$ if $x \geqq 0$. This is essentially due to the fact that parabolic schemes must make use of all the initial data at any point, as the mesh width approaches zero, and the above scheme does not. However, this interface condition is a stable one for certain hyperbolic approximations (see Ciment [1]).

5. Numerical Experiments. In this section we will present the results of numerical experiments concerning the initial boundary-value problem

$$
\begin{aligned}
& u_{t}(x, t)=u_{x x}(x, t), \quad 0<x<1, \quad 0<t<T, \\
& u(x, 0)=1 \quad \text { if } x=\frac{1}{2}, \quad u(x, 0)=0 \text { otherwise, } \\
& u(0, t)=u(1, t)=0, \quad 0 \leqq t \leqq T .
\end{aligned}
$$


We will solve (5.1) on a mesh refined in the subinterval $\left[\frac{1}{3}, \frac{2}{3}\right]$ by means of the difference scheme:

$$
\begin{aligned}
& v(x, t+k)-v(x, t)=\lambda\left(\theta \delta^{2} v(x, t+k)+(1-\theta) \delta^{2} v(x, t)\right) \\
& \text { for } x=h, 2 h, \cdots, \frac{1}{3}-h, \frac{2}{3}+h, \cdots, 1-h, t=0, k, \cdots, T-k, \\
& v(x, t+k)-v(x, t)=\lambda p^{2}\left(\theta \delta_{p}^{2} v(x, t+k)+(1-\theta) \delta_{p}^{2} v(x, t)\right) \\
& \quad \text { for } x=\frac{1}{3}+h / p, \frac{1}{3}+2 h / p, \cdots, \frac{2}{3}-h / p ; t=0, k, \cdots, T-k, \\
& v(x, 0)=1 \quad \text { if } x=\frac{1}{2}, \quad v(x, 0)=0 \text { otherwise, } \\
& v(0, t)=v(1, t)=0 \text { for } t=0, k, \cdots, T-k, T,
\end{aligned}
$$

together with interface conditions at $x=\frac{1}{3}$ and $x=\frac{2}{3}$. In the above

$$
\begin{aligned}
& \lambda=k / h^{2}, \quad p \text { is an integer } \geqq 1, \quad 0 \leqq \theta \leqq 1, \\
& \delta^{2} v(x, t)=v(x-h, t)-2 v(x, t)+v(x+h, t), \quad \text { and } \\
& \delta_{p}^{2} v(x, t)=v(x-h / p, t)-2 v(x, t)+v(x+h / p, t) .
\end{aligned}
$$

Numerical experiments were run using the scheme above with $\theta=0$ and three types of interface conditions. These results were compared to a "standard" obtained by using the above scheme with $\theta=\frac{1}{2}-h^{2} / 12 k$ (for greater accuracy) on a uniform mesh, and hence requiring no interface condition. The three interface conditions considered were

$$
\begin{aligned}
& v\left(\frac{1}{3}, t+k\right)=v\left(\frac{1}{3}, t\right)+\lambda \delta^{2} v\left(\frac{1}{3}, t\right), \\
& v\left(\frac{2}{3}, t+k\right)=v\left(\frac{2}{3}, t\right)+\lambda \delta^{2} v\left(\frac{2}{3}, t\right) .
\end{aligned}
$$

Here, we have used the difference scheme itself at the interface.

$$
\begin{aligned}
& v\left(\frac{1}{3}, t+k\right)=a_{1} v\left(\frac{1}{3}-h, t\right)+a_{2} v\left(\frac{1}{3}, t\right)+a_{3} v\left(\frac{1}{3}+h / p, t\right), \\
& v\left(\frac{2}{3}, t+k\right)=b_{1} v\left(\frac{2}{3}-h / p, t\right)+b_{2} v\left(\frac{2}{3}, t\right)+b_{3} v\left(\frac{2}{3}+h, t\right) .
\end{aligned}
$$

Consistency requires that $a_{1}=b_{3}=2 \lambda p /(p+1), a_{2}=b_{2}=(1-2 \lambda p)$, and $a_{3}=$ $b_{1}=2 \lambda p^{2} /(p+1)$. This interface condition makes use of three successive $x$-mesh points.

$$
\begin{aligned}
& v\left(\frac{1}{3}, t\right)=a_{1} v\left(\frac{1}{3}-h, t\right)+a_{2} v\left(\frac{1}{3}+h / p, t\right)+a_{3} v\left(\frac{1}{3}+2 h / p, t\right), \\
& v\left(\frac{2}{3}, t\right)=b_{1} v\left(\frac{2}{3}-2 h / p, t\right)+b_{2} v\left(\frac{2}{3}-h / p, t\right)+b_{3} v\left(\frac{2}{3}+h, t\right) .
\end{aligned}
$$

Here, consistency yields $a_{1}=b_{3}=1+p /(p+2)-2 p /(p+1), a_{2}=b_{2}=2 p /(p+1)$, $a_{3}=b_{1}=-p /(p+2)$. Notice that condition (iii) involves evaluations of $v$ which are all on the same time level.

The results of the numerical experiments, after 20 steps in the $t$ direction $(T=20 K)$ are summarized below.

\begin{tabular}{ccccccc}
\hline & $\begin{array}{c}\text { Interface } \\
\text { Condition }\end{array}$ & $h$ & $\theta$ & $p$ & $\lambda p^{2}$ & $v\left(\frac{1}{2}, T\right)$ \\
\cline { 2 - 7 } 0 & none & $1 / 24$ & .2315 & 1 & .36 & .1049 \\
1 & $($ i) & $1 / 12$ & 0 & 2 & .36 & .1045 \\
2 & (ii) & $1 / 12$ & 0 & 2 & .36 & .1098 \\
3 & (iii) & $1 / 12$ & 0 & 2 & .36 & .0987 \\
\hline
\end{tabular}


As can be seen from the above table, case 1, using the difference equation itself at the interface, gives the closest value of $v$ to the "standard" (case 0 ). This corroborates the fact that interface condition (i) has a higher order of accuracy than that of either (ii) or (iii).

6. Further Results. Theorem (2.1) can be extended to parabolic constant coefficient $(2 r+1)$-point multistep schemes, as well as to the nonparabolic two-step DuFort-Frankel approximation (see Venit [8]). In a future publication the author hopes to extend these results to get a maximum norm stability theorem for $(2 r+1)$ point initial boundary approximations to variable coefficient higher-order systems of parabolic equations. Such a theorem employing $L_{2}$ stability has been obtained by Varah [7].

Department of Mathematics

California State University at Los Angeles

Los Angeles, California 90032

1. M. Ciment, "Stable difference schemes with uneven mesh spacings," Math. Comp., v. 25,1971 , pp. $219-227$.

2. S. J. Osher, "Mesh refinements for the heat equation," SIAM J. Numer. Anal., v. 7, 1970, pp. 199-205. MR 42 \#1347.

3. S. J. OSHER, "Stability of parabolic difference schemes to certain mixed initial boundary value problems," Math. Comp., v. 26, 1972, pp. 13-39.

4. S. J. OsHER, "Systems of difference equations with general homogeneous boundary conditions," Trans. Amer. Math Soc., v. 137, 1969, pp. 177-201. MR 38 \#6259.

5. R. D. RichtMYer \& K. W. MoRTON, Difference Methods for Initial-Value Problems, 2nd ed., Interscience Tracts in Pure and Appl. Math., no. 4, Interscience, New York, 1967. MR 36 \#3515.

6. J. M. VARAH, "Maximum norm stability of difference approximations to the mixed initial boundary value problem for the heat equation," Math. Comp., v. 24, 1970, pp. 31-44. MR 41 \#4843.

7. J. M. VARAH, "Stability of difference approximations to the mixed initial boundary value problem for parabolic systems," SIAM J. Numer. Anal., v. 8, 1971, pp. 598-615.

8. S. VenIT, Mesh Refinements for Difference Approximations to Parabolic Equations, Computer Center, University of California, Berkeley, Calif., 1971.

9. O. B. WIDLUND, "Stability of parabolic difference schemes in the maximum norm," Numer. Math., v. 8, 1966, pp. 186-202. MR 33 \#5149. 